\title{
Optical Nonresonance Vector Solitons in Dispersive and Kerr Media
}

\author{
G. T. Adamashvili", N. T. Adamashvili, M. D. Peikrishvili and R. R. Koplatadze \\ Technical University of Georgia, Kostava str. 77, Tbilisi, 0179, Georgia
}

\begin{abstract}
A theory of optical nonresonance vector pulsing solitons in a Kerr media is considered. By using the multiple scale perturbative reduction method the wave equation is transformed to the coupled nonlinear Schrödinger equations. The shape of the optical nonresonance vector pulsing soliton with the difference and sum of the frequencies is presented. Explicit analytical expressions for the optical two-component vector pulsing soliton with phase modulation are obtained. It is shown that the two-component pulsing soliton in this special case can be transformed to the scalar pulsing soliton, and these waves have different shapes.
\end{abstract}

Keywords: Optical nonresonance solitons, Kerr media, two-component nonlinear waves.

\section{INTRODUCTION}

The propagation of optical waves in a medium is accompanied by different changes in their shape. The effects changing the wave form are dispersion, dissipation, and nonlinearity. These mechanisms act separately or in different combinations. Of special interest are such wave motions for which the mechanisms distorting the shape and induced by different effects exactly compensate each other. Under these conditions, nonlinear waves of stationary shape such as solitons or their different modifications are formed. The propagation of nonlinear waves of an invariable profile displays its own specific properties. In the theory of nonlinear waves they play as fundamental a role as harmonic oscillations do in the linear wave theory. The nonlinear waves of an invariable profile are one of the most important demonstrations of nonlinearity in optical systems. The conditions for the existence of nonlinear waves are different. The determination of the conditions of the existence of optical nonlinear waves of a stationary shape and the study of their features in different physical situations are among the principal problems of the nonlinear wave theory. Depending on the character of the nonlinearity, the nonresonance or resonance mechanism of the existence of nonlinear waves is realized. In the first case of nonresonant nonlinearity, which is expressed by means of the quadratic or cubic nonlinear susceptibilities, its competition with the dispersion leads to the existence of nonresonance optical solitons and pulsing solitons (breathers) [1-3]. The optical resonant nonlinear solitary waves can be excited with the help of self-induced transparency, i.e., from a coherent nonlinear resonance interaction of an optical wave with impurity atoms or semiconductor quantum dots in solids $[4,5]$.

*Address correspondence to this author at the Technical University of Georgia, Kostava str. 77, Tbilisi, 0179, Georgia; Tel: +99532222072;

Fax:+99532222072; Email: guram_adamashvili@ymail.com
Nonlinear solitary waves can be considered by a single nonlinear Schrödinger (NLS) equation for the optical onecomponent (scalar) field. Such one-component resonance and nonresonance nonlinear waves form when an optical one-component pulse propagates inside a medium while maintaining its state $[2,6]$. When this is not the case, the interaction between two field components at different polarizations or different frequencies (but possibly same polarization) has to be considered. One then has to simultaneously solve a system of coupled NLS equations. A profilepreserving solution of the coupled NLS equations is a vector pulse (soliton or pulsing soliton) because of its twocomponent configuration.

The properties of optical nonresonance vector solitons in a Kerr medium are governed by two coupled NLS equations that describe the connection between two different guided modes propagating in multi-mode optical waveguides (fibers) [7] or the coupling between two optical wave components of two distinct carrier frequencies propagating inside a single-mode waveguide [8]. In addition, in a singlemode waveguide, a single pulse also can form a vector soliton if the birefringence effects lead to a connection between its two differently polarized wave components [9].

It is of great importance to find double periodic vector soliton (vector pulsing soliton) solutions of optical nonlinear equations to provide more important information for understanding phenomena arising in different scientific fields and applications. The pulsing solitons have various interesting features that are similar to those of solitons, but unlike them, pulsing solitons can be created with relatively low input pulse energy. Therefore, pulsing solitons are easier to excite than solitons and, in addition, in some physical phenomena, pulsing solitons are more stable nonlinear waves and thus have wider potential applications in comparison to solitons (see, for example, Ref. [10]).

The theory of two-component nonresonance vector pulsing solitons with the difference and sum of the frequencies in a Kerr medium will be different from and more complex 
than the nonresonance one-component solitons and pulsing solitons [1-3] and two-component vector solitons [7-9], and a separate study will be needed.

The goal of the present work is the following: we consider the conditions of realization of the nonresonance twocomponent optical vector pulsing soliton with the difference and sum of the frequencies with a phase modulation in a Kerr medium. We explicitely determine analytic expressions for the parameters and the profile of the optical nonresonance vector pulsing soliton.

\section{BASIC EQUATIONS}

We study the propagation of optical nonresonance twocomponent vector pulsing solitons in isotropic, cubic nonlinear and second order dispersive media for linearly polarized waves with frequency $\omega \gg>T_{p}^{-1}$ and duration $T_{p}$, with the strength of the electric field $\vec{E}(z, t)=\vec{e} E(z, t)$ propagating along the positive $z$-axis, where $\vec{e}$ is the unit vector of polarization directed along the $x$-axis. Not concretizing the physical nature of the dispersive process, we describe the dependence of the dielectric function $\kappa$ by a two variables: wave vector $\vec{k}$ and frequency $\omega$ of the wave (spatial and/or temporal dispersion). We note that in optical phenomena we usually consider only temporal dispersion, but in some special physical situations, spatial dispersion can be effective too (see, for instance, Refs. [2, 3, 11, 12] and references therein).

The nonlinear wave equation has the following form $[11,12]:$

$-C^{2} \frac{\partial^{2} E}{\partial z^{2}}+\frac{\partial^{2}}{\partial t^{2}} \int \kappa\left(z_{1}, t_{1}\right) E\left(z-z_{1}, t-t_{1}\right)$

$d z_{1} d t_{1}+4 \pi \frac{\partial^{2} P}{\partial t^{2}}=0$

where $E$ is the $x$-component of the electrical field strength $\vec{E}$,

$P=\int \rho_{x x x x}\left(z_{1}, z_{2}, z_{3}, t_{1}, t_{2}, t_{3}\right) E\left(z-z_{1}, t-t_{1}\right) \times$

$E\left(z-z_{1}-z_{2}, t-t_{1}-t_{2}\right) E\left(z-z_{1}-z_{2}-z_{3}, t-t_{1}-t_{2}-t_{3}\right)$

$d z_{1} d z_{2} d z_{3} d t_{1} d t_{2} d t_{3}$

is the $x$-component of the non-resonant nonlinear polarization of the third order, $\rho_{x x x x}$ is the component of the tensor of the cubic susceptibility, $\mathrm{C}$ is the speed of light in vacuum, $\kappa(z, t)=1+4 \pi \chi_{x x x x}(z, t)$, and $\chi_{x x x x}$ is the component of the first-order susceptibility tensor.

We can transform Eq. (1) with the use of the slowly changing profiles method. In order to do this, we represent the functions $E$ as

$$
E=\sum_{l= \pm 1} \hat{E}_{l} Z_{l}
$$

where $\hat{E}_{l}$ is the slowly varying complex amplitude of the optical electric field and $Z_{l}=e^{i l(k z-\omega t)}$. To guarantee that $E(z, t)$ is a real function, we suppose that $\hat{E}_{l}=\hat{E}_{-l}^{*}$. In comparison with the carrier wave parts, the complex envelope functions $\hat{E}_{l}$ vary slowly in space and time, i.e.,

$$
\left|\frac{\partial \hat{E}_{l}}{\partial t}\right|<<\omega\left|\hat{E}_{l}\right|,\left|\frac{\partial \hat{E}_{l}}{\partial z}\right|<<k\left|\hat{E}_{l}\right| .
$$

Substituting the equations (3) into the wave equation (2), we obtain

$$
P=\sum_{l, l^{\prime}, l^{\prime \prime}} Z_{l} \tilde{\rho}_{l, l^{\prime}, l^{\prime}} \hat{E}_{l-l^{\prime}-l^{\prime \prime}} \hat{E}_{l^{\prime}} \hat{E}_{l^{\prime \prime}}
$$

where

$$
\begin{aligned}
& \tilde{\rho}_{l, l^{\prime}, l^{\prime \prime}}=\int \rho_{x x x x}\left(z_{1}, z_{2}, z_{3}, t_{1}, t_{2}, t_{3}\right) e^{-i l\left(k z_{1}-\omega t_{1}\right)} e^{-i\left(l^{\prime}+l^{\prime \prime}\right)\left(k z_{2}-\omega t_{2}\right)} . \\
& e^{-i l l^{\prime \prime}\left(k z_{3}-\omega t_{3}\right)} d z_{1} d z_{2} d z_{3} d t_{1} d t_{2} d t_{3}
\end{aligned} .
$$

Substituting the equations (3) and (4) into the wave equation (1), we obtain the dispersion relation for the nonlinear wave in the following form:

$$
C^{2} k^{2}=\omega^{2} \kappa(\omega, k)
$$

and a nonlinear wave equation:

$$
\left.\sum_{l= \pm 1} Z_{l}\left\{\begin{array}{l}
i G_{1, l} \frac{\partial \hat{E}_{l}}{\partial z}+i g_{3} \frac{\partial \hat{E}_{l}}{\partial t}+G_{2} \frac{\partial^{2} \hat{E}_{l}}{\partial z^{2}}+g_{5} \frac{\partial^{2} \hat{E}_{l}}{\partial t^{2}}- \\
g_{6} \frac{\partial^{2} \hat{E}_{l}}{\partial t \partial z} \\
-\sum_{l, l^{\prime \prime}} K_{l, l^{\prime}, l^{\prime}} \hat{E}_{l-l^{\prime}-l^{\prime}} \hat{E}_{l^{\prime}} \hat{E}_{l^{\prime \prime}}
\end{array}\right]\right\}=0
$$

where

$$
\begin{aligned}
& G_{1, l}=a \omega^{2}-2 l k C^{2}, g_{3}=-l \omega(2 \kappa+l \omega b), G_{2}=\omega_{2} c-C^{2}, \\
& g_{5}=-\left(\kappa+2 l \omega b+\omega^{2} d\right), g_{6}=l \omega(2 a+l \omega s), \\
& K_{l, l^{\prime}, l^{\prime}}=4 \pi \omega^{2} \tilde{\rho}_{l, l^{\prime}, l^{\prime \prime}}, \\
& a=\frac{\partial \kappa}{\partial(l k)}, b=\frac{\partial \kappa}{\partial(l \omega)}, c=\frac{1}{2} \frac{\partial^{2} \kappa}{\partial(l k)^{2}}, \\
& d=\frac{1}{2} \frac{\partial^{2} \kappa}{\partial(l \omega)^{2}}, s=\frac{\partial^{2} \kappa}{\partial(l k) \partial(l \omega)}, \\
& \kappa(l k, l \omega)=\int \kappa\left(z_{1}, t_{1}\right) e^{-i l\left(k z_{1}-\omega t_{1}\right)} d z_{1} d t_{1} .
\end{aligned}
$$

The function $\kappa(l k, l \omega)$ is in general complex, but we consider only the most important particular case when a wave is propagating without damping in a non-absorbing (transparent) homogeneous medium. In this case, the real part of $\kappa(l k, l \omega)$ is an even function of the frequency and 
wave number, $\kappa(l k, l \omega)=\kappa(-l k,-l \omega)$, and the imaginary part of this function is equal to zero [11, 12].

\section{NONRESONANCE VECTOR PULSING SOLITON}

For further analysis of these equations we utilize the multiple scale perturbative reduction method [13], in the limit that $\hat{E}_{l}$ is of the order $O(\varepsilon)$. Then the complex function $\hat{E}_{l}$ takes the following form:

$\hat{\mathrm{E}}_{1}(\mathrm{z}, \mathrm{t})=\sum_{\alpha=1, n=-\infty}^{\infty} \varepsilon^{\alpha} Y_{l, n} \chi_{l, n}^{(\alpha)}(\zeta, \tau)$

where

$Y_{l, n}=e^{i n\left(Q_{l, n^{n}}-\Omega_{l, n} t\right)}, \quad \zeta_{l, n}=\varepsilon Q_{l, n}\left(z-v_{l, n} t\right), \quad \tau=\varepsilon^{2} t$,

$v_{l, n}=\frac{d \Omega_{l, n}}{d Q_{l, n}}$,

$\varepsilon$ is a small parameter. This leads to a separation of the still more slowly changing functions $\chi_{l, n}^{(\alpha)}(\zeta, \tau)$ from the function $\hat{E}_{l}(z, t)$. Therefore, it follows that the quantities $\Omega_{l, n}, Q_{l, n}$, and $\chi_{l, n}^{(\alpha)}(\zeta, \tau)$ satisfy the following conditions for any $l$ and $n$ :

$\omega>>\Omega, k>>Q,\left|\frac{\partial \chi_{l, n}^{(\alpha)}}{\partial t}\right|<<\Omega\left|\chi_{l, n}^{(\alpha)}\right|,\left|\frac{\partial \chi_{l, n}^{(\alpha)}}{\partial z}\right|<<Q\left|\chi_{l, n}^{(\alpha)}\right|$.

The quantities $Q, \Omega$ and $v$ depend on 1 and n: however, the indixes 1 and $n$ will be omitted below for simplicity where their presence is clear from the context.

Obviously, for a pulse free of phase modulation, i.e., when $\hat{E}_{l}=\hat{E}_{-l}^{*}=\hat{E}$ is real, the expansion equation (8) is not valid.

Substituting Eqs. (8) into Eq. (6), we obtain

$$
\begin{aligned}
& \sum_{\substack{\alpha=1, l=1, n=-\infty}}^{\infty} \varepsilon^{\alpha} Z_{l} Y_{l, n}\left\{W_{l, n}+i \varepsilon J_{l, n} \frac{\partial}{\partial \zeta}+i \varepsilon^{2} h_{l, n} \frac{\partial}{\partial \tau}+\varepsilon^{2} \frac{\partial_{2}}{\partial \zeta^{2}}\right\} \\
& \chi_{l, n}^{(\alpha)}=\varepsilon^{3} \sum_{l} Z_{l} R_{l}\left[\begin{array}{l}
\left(\left|\chi_{l, l}^{(1)}\right|^{2}+2\left|\chi_{l,-l}^{(1)}\right|^{2}\right) \chi_{l, l}^{(1)} Y_{l, l}+\left(\left|\chi_{l,-l}^{(1)}\right|^{2}\right. \\
\left.+2\left|\chi_{l, l}^{(1)}\right|^{2}\right) \chi_{l,-l}^{(1)} Y_{l,-l}
\end{array}\right],
\end{aligned}
$$

where

$$
\begin{aligned}
& W_{l, n}=g_{3} n \Omega-G_{1, l} n Q-n^{2} G_{2} Q^{2}+g_{5} n^{2} \Omega^{2}-g_{6} n^{2} Q \Omega, \\
& J_{l, n}=g_{3} v-G_{1, l}-2 G_{2} n Q+2 n g_{5} \Omega v-g_{6} n(Q v+\Omega), \\
& h_{l, n}=g_{3}+2 g_{5} n \Omega-g_{6} n Q, \\
& H_{l, n}=Q^{2}\left(G_{2}-g_{5} v^{2}+g_{6} n Q\right), \\
& R_{l}=4 \pi \omega^{2}\left(\tilde{\rho}_{l, l, l}+\tilde{\rho}_{l, l,-l}+\tilde{\rho}_{l,-l, l}\right) .
\end{aligned}
$$

To find the form of the functions $\chi_{l, n}^{(\alpha)}(\zeta, \tau)$, we equate to zero the terms that have the same order of $\varepsilon$. This results in a system of equations.

In the first order in $\varepsilon$, we obtain the equations

$\sum_{\substack{l= \pm 1, n= \pm 1}} Z_{l} Y_{l, n} W_{l, n} \chi_{l, n}^{(1)}=0$

We have to note that we shall be interested in localized solitary waves which vanish as $t \rightarrow \pm \infty$. Hence, according to Eq.(11), only the following functions of $\chi_{l, n}^{(1)}$ can differ from zero: $\chi_{+, \pm 1}^{(1)}$. From the equation $\hat{E}_{l}=\hat{E}_{-l}^{*}$, we obtain $\chi_{e, n}^{(\alpha)^{*}}=\chi_{-l,-n}^{(\alpha)}$.

The relations between the quantities $\Omega$ and $Q$ are obtained from Eq. (11) and have the following form:

$g_{3} n \Omega-G_{1, l} n Q-G_{2} Q^{2}+g_{5} \Omega^{2}-g_{6} Q \Omega=0$.

To second order in $\varepsilon$ we have for the functions $f_{l, n}^{(2)}(\zeta, \tau)$ the following equations:

$\sum_{\substack{l= \pm 1, n=-\infty}} Z_{l} Y_{l, n}\left(W_{l, n} \chi_{l, n}^{(2)}+i \varepsilon J_{l, n} \frac{\partial \chi_{l, n}^{(1)}}{\partial \zeta}\right)=0$.

Substituting Eqs. (12) into Eq. (10), we arrive at the following equation: $J_{ \pm 1, \pm 1}=J_{ \pm 1, \mp 1}=0$. From Eqs. (9) and (13), to third order in $\varepsilon$, we obtain the following nonlinear equation:

$$
\begin{aligned}
& i \frac{\partial \chi_{+1, \pm 1}^{(1)}}{\partial \tau}-\frac{H_{+1, \pm 1}}{h_{+1, \pm 1}} \frac{\partial^{2} \chi_{+1, \pm 1}^{(1)}}{\partial \zeta^{2}}-\frac{R^{2}}{2 h_{+1, \pm 1}} \\
& {\left[\left|\chi_{+1, \pm 1}^{(1)}\right|^{2}+\left(1-\frac{\Omega_{\mp}}{\Omega_{ \pm}}\right)\left|\chi_{+1, \mp 1}^{(1)}\right|^{2}\right] \chi_{+1, \pm 1}^{(1)}=0 .}
\end{aligned}
$$

From Eq. (14), we derive two coupled NLS equations for the functions $u=\varepsilon \chi_{+1,+1}^{(1)}$ and $w=\varepsilon \chi_{+1,-1}^{(1)}$ that determine the connection between the two components of the pulse,

$$
\begin{aligned}
& i\left(\frac{\partial u}{\partial t}+v_{1} \frac{\partial u}{\partial z}\right)+p_{1} \frac{\partial^{2} u}{\partial z^{2}}+q_{1}\left|u^{2}\right| u+r_{1}\left|w^{2}\right| u=0, \\
& i\left(\frac{\partial w}{\partial t}+v_{2} \frac{\partial w}{\partial z}\right)+p_{2} \frac{\partial^{2} w}{\partial z^{2}}+q_{2}\left|w^{2}\right| w+r_{2}\left|u^{2}\right| w=0,
\end{aligned}
$$

where

$$
\begin{aligned}
& v_{1}=v_{+1,+1}, \quad v_{2}=v_{+1,-1}, \\
& p_{1}=\frac{H_{+1,+1}}{-h_{+1,+1} Q^{2}}, \quad p_{1}=\frac{H_{+1,-1}}{-h_{+1,-1} Q^{2}},
\end{aligned}
$$


$q_{1}=\frac{R^{2}}{-2 h_{+1,+1}}, \quad q_{2}=\frac{R^{2}}{-2 h_{+1,-1}}$,

$r_{1}=q_{1}\left(1-\frac{\Omega_{-1}}{\Omega_{+1}}\right), \quad r_{2}=q_{2}\left(1-\frac{\Omega_{+1}}{\Omega_{-1}}\right)$,

$\Omega_{+1}=\Omega_{l= \pm 1, n= \pm 1}, \quad \Omega_{-1}=\Omega_{l= \pm 1, n=\mp 1}, \quad Q_{+1}=Q_{l= \pm 1, n= \pm 1}$,

$Q_{-1}=Q_{l= \pm 1, n=\mp 1}$.

The nonlinear equations (15) determine the slowly varying functions $u$ and $w$, where $u$ represents the wave which oscillates with the frequency $\omega+\Omega_{+1}$ and $w$ describes the wave with frequency $\omega-\Omega_{-1}$. The nonlinear connection between the two functions $u$ and $w$ is determined by the terms $r_{1}\left|w^{2}\right| u$ and $r_{2}\left|u^{2}\right| w$. Our next task is to look at the interaction between the two field components $u$ and $w$ which have different frequencies but same polarizations, and solve simultaneously a system of coupled NLS equations (15). A form-invariant solution of the equations (15) is a vector soliton due to its two-component configuration.

In order to ensure the stable shape of the soliton, the electric field envelope function $\hat{E}_{l}(z, t)$ has to depend on the space and time coordinate only through the new coordinate $\xi=t-\frac{z}{V_{0}}$, where $V_{0}$ is the speed of the vector pulse. We search for the steady-state solutions for the strength of the electrical field of the pulse of Eqs. (15) for the slowly varying functions $u$ and $w$, which will be of the form

$u(z, t)=A_{1} e^{i \Phi_{1}} S(\xi), w(z, t)=A_{2} e^{i \Phi_{2}} S(\xi)$

where $\Phi_{1,2}=k_{1,2} z-\omega_{1,2} t$ are the phase functions and $A_{1,2}, k_{1,2}$ and $\omega_{1,2}$ are real constants. Compared to the oscillations of the pulse, the functions $e^{i \Phi_{1,2}(z, t)}$ vary very slowly, from which it follows that the inequalities

$k_{1,2}<<Q_{ \pm 1}, \omega_{1,2}<<\Omega_{1,2}$

are satisfied.

Substituting Eqs. (17) into Eqs. (15), we obtain the nonlinear ordinary differential equation for the function $S(\xi)$,

$\left(\frac{d S}{d \xi}\right)^{2}=T_{p}^{-2} S^{2}-b^{2} S^{4}$

and the equations for the quantities $A_{1,2}$ and $\omega_{1,2}$

$$
\begin{aligned}
& A_{1}^{2}=\frac{p_{1} q_{2}-p_{2} r_{1}}{p_{2} q_{1}-p_{1} r_{2}} A_{2}^{2} \\
& \omega_{1}=\frac{p_{1}}{p_{2}} \omega_{2}+\frac{V_{0}\left(p_{2}^{2}-p_{1}^{2}\right)+v_{2}^{2} p_{1}^{2}-v_{1}^{2} p_{2}^{2}}{4 p_{1} p_{2}^{2}},
\end{aligned}
$$

where

$b^{2}=V_{0}^{2} \frac{A_{1}^{2} q_{1}+A_{2}^{2} r_{1}}{2 p_{1}}$

After integration of the equation (19), we have

$S(\xi)=\frac{1}{b T_{p}} \sec h \frac{\xi}{T_{p}}$

This is a soliton ( $2 \pi$ pulse) solution of the wave equation [4].

Consequently, we find that the components $u$ and $w$ are hyperbolic secants $S(\xi)$ with different amplitudes $A_{1,2}$ and phase functions $\Phi_{1,2}$.

Substituting the function $S(\xi)$ [Eq. (21)] into Eqs. (3), (8) and (17), we obtain for the strength of the electric field $E(z, t)$ the two-component vector pulsing soliton solution of the Maxwell equation (1) in the form

$$
\begin{aligned}
& E(z, t)=\frac{2}{q T_{p}} \sec h\left[\frac{1}{T_{p}}\left(t-\frac{z}{V_{0}}\right)\right]\left\{\left(\Omega_{+1}+\omega_{1}\right) \sin \right. \\
& {\left[\left(k+Q_{+1}+k_{1}\right) z-\left(\omega+\Omega_{+1}+\omega_{1}\right) t\right]} \\
& \left.-\left(\Omega_{-1}-\omega_{2}\right) \sqrt{\frac{p_{1} q_{2}-p_{2} r_{1}}{p_{2} q_{1}-p_{1} r_{2}}} \sin \left[\begin{array}{l}
\left(k-Q_{-1}+k_{2}\right) z \\
-\left(\omega-\Omega_{-1}+\omega_{2}\right) t
\end{array}\right]\right\}
\end{aligned}
$$

where

$$
\begin{aligned}
& T_{p}^{-2}=V_{0}^{2} \frac{v_{1} k_{1}+k_{1}^{2} p_{1}-\omega_{1}}{p_{1}}, q^{-2}=\frac{2}{V_{0}^{2}} \frac{p_{1} q_{2}-p_{2} r_{1}}{q_{1} q_{2}-r_{1} r_{2}}, \\
& k_{1,2}=\frac{V_{0}-v_{1,2}}{2 p_{1,2}}
\end{aligned}
$$

\section{CONCLUSION}

We demonstrated that in cubic nonlinear and second order (spatially and/or temporally) dispersive media, an optical nonresonance two-component vector pulsing soliton can arise. The explicit expressions for the parameters and profile of the optical nonresonance vector pulsing soliton are given by Eqs. (7), (16), (20), (22) and (23). The dispersion law and the connection between the quantities $\Omega_{ \pm 1}$ and $Q_{ \pm 1}$ are given by Eqs. (5) and (12), respectively.

In Eq. (22), the functions $\sin \left[\left(k+Q_{+1}+k_{1}\right) z-\left(\omega+\Omega_{+1}\right.\right.$ $\left.\left.+\omega_{1}\right) t\right]$ and $\sin \left[\left(k-Q_{-1}+k_{2}\right) z-\left(\omega-\Omega_{-1}+\omega_{2}\right) t\right]$ point to the formation of double periodic oscillations with time and position coordinates relative to the frequency and wave number of the carrier wave $(\omega, k)$, with characteristic parameters $\left(\omega+\Omega_{+1}, k+Q_{+1}\right)$ and $\left(\omega-\Omega_{-1}, k-Q_{-1}\right)$. Taking into account Eq. (18), the soliton solution (21) for $S(\xi)$ is transformed into a two-component vector pulsing soliton solution (22) for the strength of the electric field of the opti- 


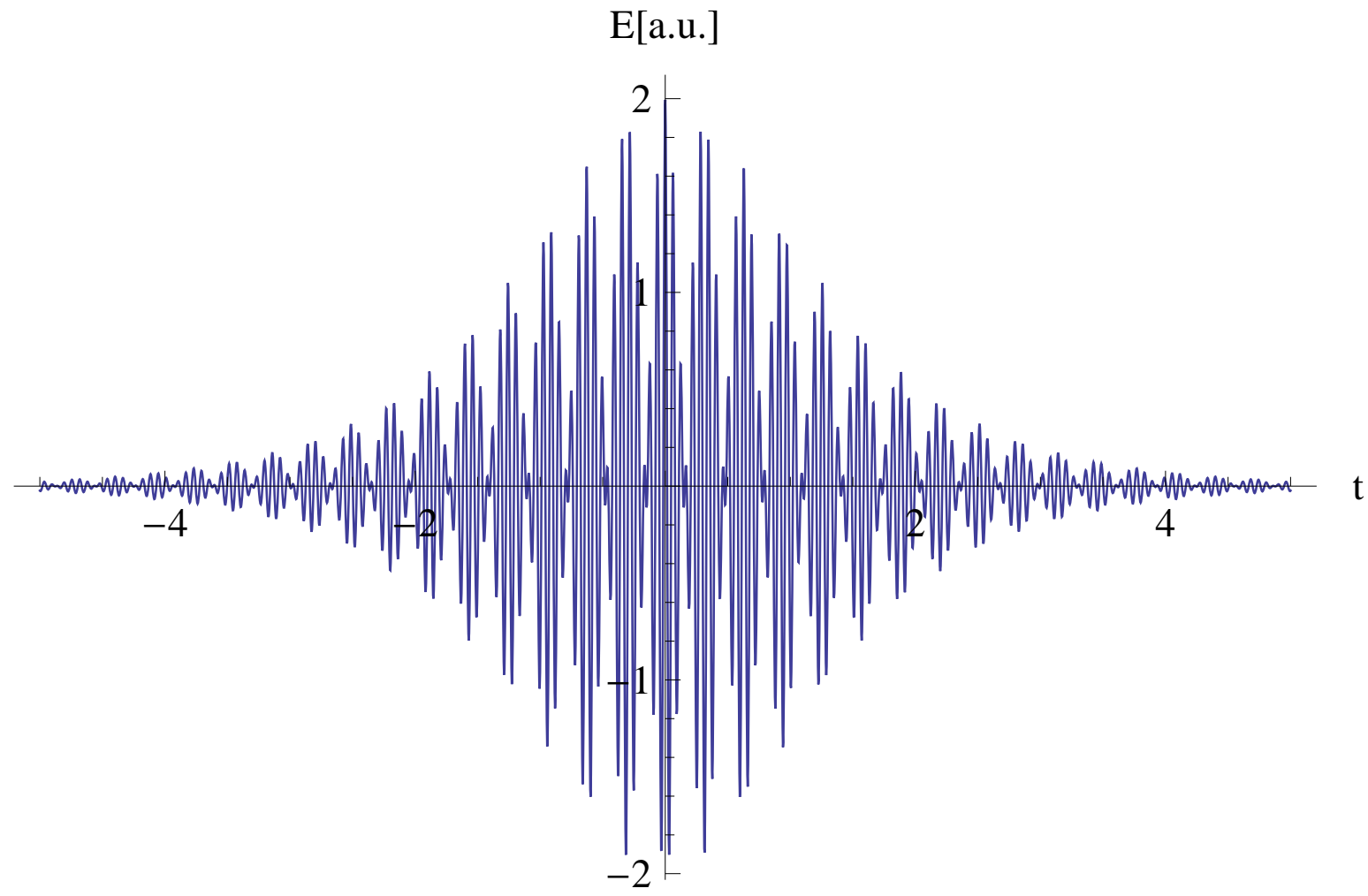

Fig. (1). The $x$-component of the strength of the electrical field $E(0, t)$ of the two-component vector pulsing soliton is shown for a fixed value of $z$. The nonlinear pulse oscillates with the difference $\omega-\Omega_{-1}$ and sum $\omega+\Omega_{+1}$ of the frequencies along the $t$-axis.

cal pulse $E(z, t)$. Eq. (22) is a double periodic solution of Eq. (1) which, like a one-component soliton, breather and vector soliton, loses no energy during propagation through the medium.

A vector pulsing soliton is an absolutely different nonlinear wave in comparison with nonresonance one-component solitons and breathers [1-3, 5, 6] and vector solitons [7-9] which have been investigated up to now. The vector pulsing soliton is a complex nonlinear wave which consists of two coupled pulsing solitons with different frequencies of oscillation and the same polarizations (along the $x$-axis), which in the process of propagation exchange the energy between each other. In the particular case when the interaction between these coupled pulsing solitons is very weak $\left(r_{1,2} \rightarrow 0\right.$ in equations (15)), the exchange energy is also very small. In the limiting case when $r_{1}=r_{2}=0$, where the interaction between the coupled pulsing solitons is equal to zero (the coupled NLS equations (15) will be disconnected), the pulsing solitons are propagating independently of each other. Under this condition, we can excite only one scalar pulse (onecomponent pulse) which has been investigated in Refs. $[2,3,5,6]$.

A plot of the two-component vector pulsing soliton Eq. (22) is shown in Fig. (1). for a fixed value of the $z$ coordinate. We assume that the quantities $\omega / \Omega_{ \pm 1}$ are of the order $10^{3}$. In the optical region of the spectrum, $\omega$ is of the order
$10^{15} s^{-1}$ and with a typical numerical values for the pulse width in glass $T_{p}=100$ ps [1], the condition $\Omega_{ \pm 1} T_{p}>>1$ is fulfilled.

The one-component pulsing soliton is a special case of the vector pulsing soliton Eq. (22). The shape of the onecomponent pulsing soliton with the same values of the parameters as for the vector pulsing soliton is presented in Fig. 2. It is obvious that the profile of the two-component vector pulsing soliton (Fig. 1) differs from the profile of the onecomponent pulsing soliton (Fig. 2).

The conditions of observability of the predicted beating effect in the carrier wave can be realized analogously to the experimental investigations of few-cycle pulses with durations of only a few periods of the wave (see, for instance, Ref. [14] and references therein).

The results of this theoretical study of nonresonance vector pulsing solitons, together with those obtained in Refs. [1$3,5,6]$ for one-component solitons and breathers and in Refs. [7-9] for vector solitons, provide a more complete physical description of the propagation of non-resonance nonlinear waves in dispersive and Kerr media.

The presented results will stimulate research of multicomponent nonresonance vector pulsing pulses in Kerr media using, for example, a more complex form of Eq. (8).

We would like to emphasize that our findings are quite general and can be transformed for second order (spatially 
E a.u.

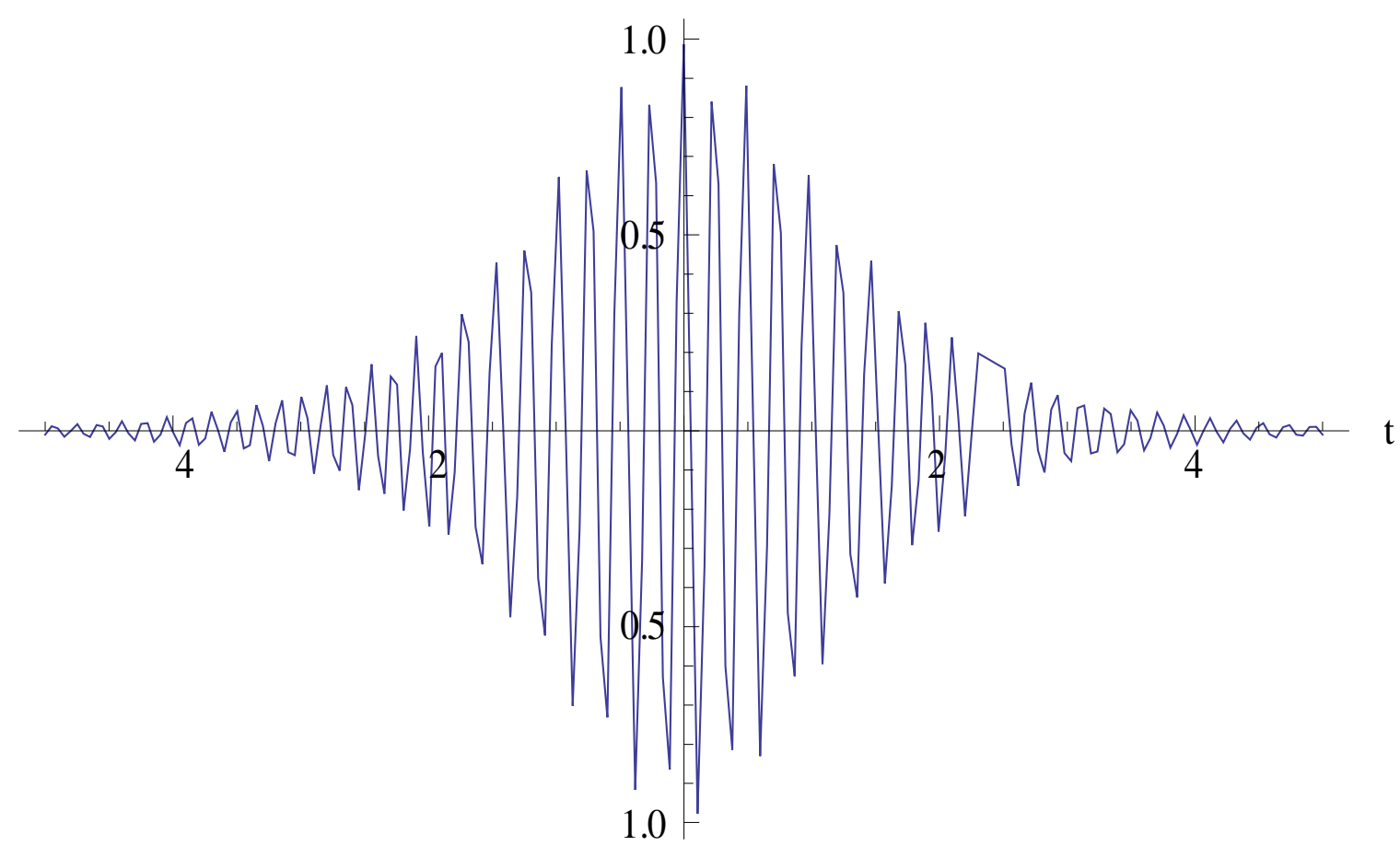

Fig (2). The $x$-component of the strength of the electrical field $E(0, t)$ of the one-component scalar pulsing soliton is shown for a fixed value of $z$.

and/or temporally) dispersive and noncentrosymmetric crystals with quadratic susceptibility.

\section{REFERENCES}

[1] Sauter EG. Nonlinear Optics. Wiley: New York 1996.

[2] Adamashvili GT, Kaup DJ. Optical nonresonance and two-photon resonance solitons in anisotropic media. Phys Rev (E) 2004; 70: 066616-12.

[3] Adamashvili GT, Maradudin AA. Nonresonance solitons in nonlinear and dispersive media. Phys Rev (E) 1997; 55: 7712-9.

[4] Allen L, Eberly JN. Optical Resonance and Two Level Atoms. Wiley-Interscience Publ 1975.

[5] Adamashvili GT, Knorr A. Optical solitons in semiconductor quantum dots. Opt Lett 2006; 31: 74-6.

[6] Adamashvili GT. Optical solitons in anisotropic media. Phys Rev (E) 2004; 69: 026608-12.
[7] Crosignani B, Di Porto P. Soliton propagation in multimode optical fibers. Opt Lett 1981; 6: 329-30.

[8] Agrawal GP. Nonlinear Fiber Optics, 3rd ed. Academic: San Diego 2001.

[9] Menyuk CR. Pulse propagation in an elliptically birefringent Kerr medium. IEEE J Quant Electron 1989; 25: 2674.

[10] Chen M, Kaup DJ, Malomed BA. Three-wave solitons and continuous waves in media with competing quadratic and cubic nonlinearities. Phys Rev (E) 2004; 69: 056605-9.

[11] Landau LD, Lifshitz EM. Electrodynamics of Continuous Media. Pergamon Press: New York 1984.

[12] Vinogradova MB, Rudenko OV, Suhorukov AP. Theoria voln. Nauka: Moscow 1990

[13] Taniuti T, Iajima N. Perturbation Method for a Nonlinear Wave Modulation. III. J Math Phys 1973; 14: 1389.

[14] Rozanov NN. Maxwell-Drude-Bloch dissipative few-cycle optical solitons. Phys Rev (A) 2010; 81:043815-17.

Received: July 25, 2013

(C) Adamashvili et al.; Licensee Bentham Open.

This is an open access article licensed under the terms of the Creative Commons Attribution Non-Commercial License (http://creativecommons.org/licenses/by-nc/3.0/) which permits unrestricted, non-commercial use, distribution and reproduction in any medium, provided the work is properly cited. 